\title{
Determinants and consequences of malnutrition in hospitalized patients at a Norwegian University Hospital
}

\author{
Marte A. Trollebo ${ }^{1}$, Ingrid Revheim ${ }^{2}$, Hanne Rosendahl-Riise ${ }^{3}$, Mette H. Morken ${ }^{4}$, \\ Randi J. Tangvik ${ }^{1,2}$ and Jutta Dierkes ${ }^{3,5}$ \\ ${ }^{1}$ University Bergen, Dept. of Clinical Medicine, Centre for Nutrition, Bergen, Norway, \\ ${ }^{2}$ Dept. of Research and Development, Haukeland University Hospital, Bergen, Norway, \\ ${ }^{3}$ University Bergen, Dept. of Clinical Medicine, Mohn nutrition research laboratory, Bergen, Norway, \\ ${ }^{4}$ Dept. of Clinical Nutrition, Haukeland University Hospital, Bergen, Norway and \\ ${ }^{5}$ Dept. of Laboratory Medicine and Pathology, Haukeland University Hospital, Bergen, Norway
}

\begin{abstract}
Background:

Malnutrition is a serious condition that is frequent in hospitalized patients even in countries with high healthcare standards, and may affect up to $30 \%$ of all hospitalized patients in tertiary hospitals. Malnutrition or risk of malnutrition (hereafter referred to as malnutrition) strongly relate to clinical outcomes and mortality, even after adjustment for age and co-morbidities. There is a great need for improved methods for detecting and treating malnutrition in this population.
\end{abstract}

\section{Objective:}

Investigate factors that are associated with malnutrition at hospitalization, and provide data on specific nutrient deficiencies that are associated with malnutrition. Analyse the association of malnutrition with nutritional biomarkers, quality of life, disease-related functions and re-hospitalization, morbidity and mortality.

\section{Design:}

The study is s a prospective case-control study including patients from different Departments of a tertiary hospital (Haukeland University Hospital, Bergen, Norway). As part of the patients security programme, all hospitalized patients are routinely screened for nutritional risk using NRS-2002. The result allocates the patient to either the case- or the control group. Included patients undergo extensive examinations; standardized assessments of quality of life, socio-economic status, lifestyle habits, dietary habits and food intake, and anthropometric measurements (mid-upper arm circumference, waist circumference and body composition by bioelectrical impedance analysis). Blood and urine samples are collected to assess nutritional biomarkers related to malnutrition.

Results:

The study recruitment is ongoing and up to April 2019, 220 patients have been recruited $(\mathrm{n}=90(41 \%)$ at nutritional risk, $\mathrm{n}=130$ $(59 \%)$ not at nutritional risk. Most of the patients are recruited from the Department of Heart Disease, $n=69(31 \%)$ and Thoracic Medicine, $\mathrm{n}=98(45 \%)$. The recruitment of patients faces many challenges, including a high degree of non-participation (about one in four patients), and a high degree of misclassification (patients falsely categorized as 'not at nutritional risk').

\section{Conclusion:}

The study is unique due to the integration of patient-derived data on quality of life, food intake, and socio-economic factors with multiple and complementary nutritional biomarkers. A first result from the recruitment period is the need for specification of the specificity and sensitivity of screening tools for malnutrition in hospitals under realistic circumstances. This should ideally be tested in a multicentre study

\section{Conflict of Interest}

There is no conflict of interest. 